\title{
A spherical Hopfield model
}

\author{
D. Bollé ${ }^{a}$, Th.M. Nieuwenhuizen ${ }^{b}$, I. Pérez Castillo ${ }^{a}$, T. Verbeiren ${ }^{a}$
}

October 29, 2018

\author{
${ }^{a}$ Instituut voor Theoretische Fysica, Katholieke Universiteit Leuven, \\ Celestijnenlaan 200 D, B-3001 Leuven, Belgium \\ ${ }^{b}$ Institute for Theoretical Physics, Valckenierstraat 65, 1018 XE Amsterdam, \\ The Netherlands
}

\begin{abstract}
We introduce a spherical Hopfield-type neural network involving neurons and patterns that are continuous variables. We study both the thermodynamics and dynamics of this model. In order to have a retrieval phase a quartic term is added to the Hamiltonian. The thermodynamics of the model is exactly solvable and the results are replica symmetric. A Langevin dynamics leads to a closed set of equations for the order parameters and effective correlation and response function typical for neural networks. The stationary limit corresponds to the thermodynamic results. Numerical calculations illustrate our findings.
\end{abstract}

\section{Introduction}

In general, the introduction of continuous versions of discrete spin models in statistical mechanics has always been welcomed. Since the work of Kac and Berlin [1] the spherical model has lead to a better understanding of a lot of basic phenomena due to the fact that they simplify the mathematical calculations considerably. In spin glass research there is no doubt that an important role has been played by the spherical p-spin spin glass model [2] 3]. Since then it has been quite remarkable to see how a mean-field model with spherical spins resembles the results of realistic structural glasses (see, e.g., the many references in [4]).

In neural network theory the Hopfield model is by now a well known classic found in many textbooks (e.g., [5], 6]) to study storage and retrieval in associative memory 
problems. Strangely enough, a spherical version of it has not yet been studied in the literature. One of the reasons might be that by relaxing the neurons from Isingtype to spherical-type variables the retrieval phase is absent, i.e. one of the most important properties of this model is lost.

In this work we show that this problem has an easy and elegant solution. By introducing a quartic potential term in the Hopfield Hamiltonian such that the neurons stay spherical the retrieval phase is recovered. In this way we obtain a simplified version of the Hopfield model with an exactly solvable thermodynamics leading to marginally replica symmetric results. A phase diagram is obtained with an explicit solution for the spin-glass retrieval 'transition' line, showing no re-entrance. Moreover, the region of global stability for the retrieval solutions is larger than the corresponding region in the standard Hopfield model.

Next, we study the relaxational Langevin dynamics of this model leading to a closed set of equations for the order parameters and effective correlation and response functions, typical for neural networks. These equations are similar to those of other models with continuous spins (see, e.g, 7] and references therein). We discuss the evolution of the overlap order parameter in the retrieval phase. The stationary limit of the dynamics is found to correspond to the thermodynamic results. The numerical solution of the dynamics illustrates this behaviour.

The rest of the paper is organized as follows. In section 2 we introduce the model. Section 3 contains the thermodynamical properties with the temperature-capacity phase diagram. In section 4 we study the dynamics showing the evolution of the retrieval overlap. Finally, section 5 summarizes our conclusions.

\section{A spherical Hopfield model}

The Hopfield model [8] is defined through the following mean-field Ising-type Hamiltonian

$$
\mathcal{H}(\{\boldsymbol{\sigma}\})=-\frac{1}{2} \sum_{i \neq j=1}^{N} J_{i j} \sigma_{i} \sigma_{j},
$$

where the couplings $J_{i j}$ are related with the information one wants to store in the network through the Hebbian rule

$$
J_{i j}=\frac{1}{N} \sum_{\mu=1}^{p} \xi_{i}^{\mu} \xi_{j}^{\mu},
$$

with $p=\alpha N$, where $\alpha$ is the loading capacity of the network. In this work, the $p$ patterns $\xi_{i}^{\mu}$ are choosen to be a collection of continuous indepnedent identical random 
variables (i.i.d.r.v.) with respect to $i$ and $\mu$ and drawn from a gaussian distribution

$$
P\left(\xi_{i}^{\mu}\right)=\frac{1}{\sqrt{2 \pi}} \exp \left[-\frac{\left(\xi_{i}^{\mu}\right)^{2}}{2}\right]
$$

and the neurons (spins) are also taken to be continuous and to satisfy the spherical constraint

$$
\sum_{i=1}^{N} \sigma_{i}^{2}=N
$$

As we will show explicitly below this setup of the spherical Hopfield model does not allow for a retrieval phase. Therefore, we add the following term to the Hamiltonian (11)

$$
-\frac{u_{0}}{4} \sum_{i, j, k, l} J_{i j k l} \sigma_{i} \sigma_{j} \sigma_{k} \sigma_{l}, \quad J_{i j k l}=\frac{1}{N^{3}} \sum_{\mu=1}^{p} \xi_{i}^{\mu} \xi_{j}^{\mu} \xi_{k}^{\mu} \xi_{l}^{\mu},
$$

which is a quartic term in the order parameter $m$ characterizing the retrieval phase

$$
m^{\mu}=\frac{1}{N} \sum_{i=1}^{N} \xi_{i}^{\mu} \sigma_{i}
$$

The introduction of this quartic term turns out to contribute macroscopically to the condensed part of the free energy but it only leads to sub-extensive contributions to the noise produced by the non-condensed patterns and to a sub-extensive contribution coming from the diagonal terms, as we now discuss in the following section.

\section{Thermodynamic and retrieval properties.}

We apply the standard replica technique [9] in order to study the thermodynamical properties of the model defined above. Starting from the Hamiltonian

$$
\mathcal{H}(\boldsymbol{\sigma})=-\frac{1}{2} \sum_{i \neq j} J_{i j} \sigma_{i} \sigma_{j}-\frac{u_{0}}{4} \sum_{i, j, k, l} J_{i j k l} \sigma_{i} \sigma_{j} \sigma_{k} \sigma_{l}
$$

and assuming, for simplicity, one condensed pattern, the replicated free energy per site becomes

$$
\beta f=\lim _{N \rightarrow \infty} \lim _{n \rightarrow 0} \frac{-1}{N n} \int_{-\infty}^{\infty} \prod_{\alpha=1}^{n} d m^{\alpha} \int_{-\infty}^{\infty} \prod_{\alpha \neq \beta} d q_{\alpha \beta} \int_{-i \infty}^{i \infty} \prod_{\alpha=1}^{n} \frac{d u_{\alpha}}{4 \pi i} \int_{-i \infty}^{i \infty} \prod_{\alpha \neq \beta} \frac{d \widehat{q}_{\alpha \beta}}{4 \pi i / N} \mathrm{e}^{-N g}
$$


with

$$
\begin{aligned}
& g=-\frac{\beta}{2} \sum_{\alpha=1}^{n} m_{\alpha}^{2}-\frac{u_{0} \beta}{4} \sum_{\alpha=1}^{n} m_{\alpha}^{4}+\frac{n \alpha \beta}{2}+\frac{1}{2} \sum_{\alpha, \beta=1}^{n} m_{\alpha}\left(q^{-1}\right)_{\alpha \beta} m_{\beta} \\
& -\alpha n \ln 2 \pi+\frac{\alpha}{2} \ln \operatorname{det}(\mathbb{I}-\beta \mathbf{q})-\frac{n}{2} \ln 2 \pi+\frac{1}{2} \ln \operatorname{det} \widehat{\mathbf{Q}}-\frac{1}{2} \sum_{\alpha, \beta=1}^{n} \widehat{Q}_{\alpha \beta} q_{\alpha \beta} .
\end{aligned}
$$

In this expression

$$
m_{\alpha}^{1}=\frac{1}{N} \sum_{i=1}^{N} \xi_{i}^{1} \sigma_{i}^{\alpha}, \quad q_{\alpha \beta}=\frac{1}{N} \sum_{i=1}^{N} \sigma_{i}^{\alpha} \sigma_{i}^{\beta}, \quad \alpha \neq \beta
$$

are the retrieval overlap, respectively the neuron (spin) overlap order parameters, $\widehat{q}_{\alpha \beta}$ are conjugate variables and

$$
\widehat{Q}_{\alpha \beta}=u_{\alpha} \delta_{\alpha \beta}-\widehat{q}_{\alpha \beta}\left(1-\delta_{\alpha \beta}\right) .
$$

Within a K-th order Parisi replica breaking scheme we assume that the spin overlap matrix has the following (ultrametric) structure

$$
q_{\alpha \beta}=q_{i}, \quad \text { if } I\left(\alpha / m_{i}\right) \neq I\left(\beta / m_{i}\right) \text { and } I\left(\alpha / m_{i+1}\right)=I\left(\beta / m_{i+1}\right)
$$

with $\left\{q_{i}\right\}_{i=0, \ldots, K}$ a set of real numbers and $\left\{m_{i}\right\}_{i=1, \ldots, K}$ a set of integers such that $m_{i+1} / m_{i}$ is an integer $\left(m_{0}=n, m_{K+1}=1\right)$, and we introduce the inverse of the Parisi function

$$
x(q)=n+\sum_{i=0}^{K}\left(m_{i+1}-m_{i}\right) \Theta\left(q-q_{i}\right) .
$$

In the limit $K \rightarrow \infty, q_{0} \rightarrow 0$ and $q_{K} \rightarrow q_{M}$ such that the free energy per site reads in the limit $n \rightarrow 0, N \rightarrow \infty$

$$
\begin{gathered}
\beta f[x(q), m]=-\frac{\beta}{2} m^{2}-\frac{u_{0} \beta}{4} m^{4}+\frac{\alpha \beta}{2}-\left(\alpha+\frac{1}{2}\right) \ln 2 \pi-\frac{1}{2}+\frac{\alpha}{2}\left[\ln \left[1-\beta\left(1-q_{M}\right)\right]\right. \\
\left.-\beta \int_{0}^{q_{M}} \frac{d q}{1-\beta \int_{q}^{1} x\left(q^{\prime}\right) d q^{\prime}}\right]-\frac{1}{2}\left[\int_{0}^{q_{M}} \frac{d q}{\int_{q}^{1} x\left(q^{\prime}\right) d q^{\prime}}+\ln \left(1-q_{M}\right)-\frac{m^{2}}{\int_{0}^{1} x(q) d q}\right],
\end{gathered}
$$

where $f$ has to be extremized with respect to $x(q)$ and $m$. Taking, in general, $x(q)$ to be a piece-wise continuous function it is straightforward to show following [2, 10] that the solution for $x(q)$ reads

$$
x(q)=\Theta\left(q-q_{M}\right)
$$


and, hence, the replica solution is exact. The free energy then reads

$$
\begin{aligned}
\beta f=\operatorname{extr}_{m, q}\left\{-\frac{\beta}{2} m^{2}-\frac{u_{0} \beta}{4} m^{4}+\frac{\alpha \beta}{2}-\left(\alpha+\frac{1}{2}\right) \ln 2 \pi-\frac{1}{2}\right. \\
\left.+\frac{\alpha}{2}\left[\ln [1-\beta(1-q)]-\frac{\beta q}{1-\beta(1-q)}\right]-\frac{1}{2}\left[\ln [1-q]+\frac{q-m^{2}}{1-q}\right]\right\}
\end{aligned}
$$

with $q_{M}=q$, the Edwards-Anderson orderparameter and $m^{1}=m$.

In agreement with this, one can show by investigating the stability against replica symmetry breaking fluctuations that the replicon eigenvalue is zero and, hence, the replica symmetric result is marginally stable.

The saddle-point equations then become simple algebraic equations for $q$ and $m$

$$
m\left(u_{0} m^{2}-\frac{1-\chi}{\chi}\right)=0, \quad \frac{q-m^{2}}{\chi^{2}}=\frac{\alpha q}{(1-\chi)^{2}}
$$

with $\chi=\beta(1-q)$ the susceptibility. We immediately remark that when we remove the quartic interaction by setting $u_{0}=0$ there exist no solution with $m \neq 0$ and, hence, there is no retrieval possible.

Henceforth, we take $u_{0}=1$. The corresponding $T-\alpha$ phase diagram is shown in Fig. 1. Let us compare this result with the phase diagram of the standard Hopfield

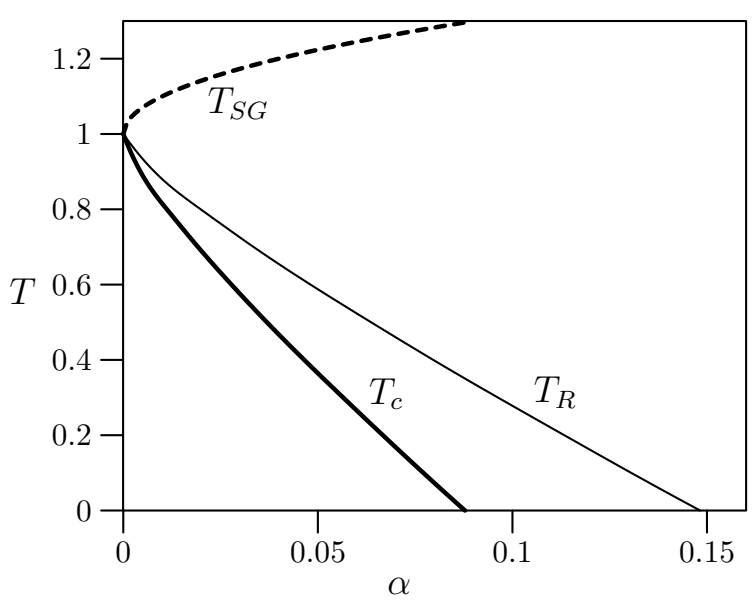

Figure 1: $T-\alpha$ phase diagram for the spherical Hopfield model. Full (dashed) lines indicate discontinuous (continuous) transitions: $T_{S G}$ describes the spin glass transition and $T_{R}$ (19)-(20) indicates the border of existense of the retrieval solutions, $T_{c}$ denotes the thermodynamic transition below which the retrieval states are global minima of the free energy.

model calculated in a replica symmetric approximation [5, 11]. Again we have three 
phases. For temperatures above the broken line $T_{S G}$, there exist paramagnetic solutions characterized by $m=q=0$, while below the broken line, spin glass solutions, $m=0$ but $q \neq 0$, exist. The transition between the paramagnetic and the spin glass phase is continuous and the line $T_{S G}$ separating the two phases is easily computed

$$
T_{S G}(\alpha)=1+\sqrt{\alpha}
$$

as in the standard Hopfield model. Next, below the thin full line $T_{R}$, (locally stable) retrieval solutions, $m \neq 0$ and $q \neq 0$, appear. The transition between the spinglass and the retrieval phase is, however, a discontinous transition. Using then the information that the values of both the order parameters $q$ and $m$ jump when passing this line $T_{R}$, it is possible to find an analytical expression for the latter. Writing the equations (17) as two polynomials in $q$ and $m$, a jump means that complex roots of these two polynomials become real at the same time for all values of $\alpha, T$ obtained when crossing $T_{R}$. After some algebra this leads to

$$
\alpha_{R-S G}(\beta)=\frac{2}{81 \beta^{5}}\left[8(\beta-1)^{2} \beta^{2}(7 \beta-16)+\operatorname{sgn}(\Delta) \frac{\Sigma}{\sqrt[3]{|\Delta|}}+\operatorname{sgn}(\Delta) \sqrt[3]{|\Delta|}\right]
$$

with

$$
\begin{aligned}
\Delta & =81 \sqrt{3}(\beta-1)^{9 / 2} \beta^{17 / 2}(|125 \beta-128|)^{3 / 2}-\Delta_{2} \\
\Delta_{2} & =(\beta-1)^{5} \beta^{6}\{-2097152+\beta(4849664+\beta[-3459072+625 \beta(1088+25 \beta)])\} \\
\Sigma & =(\beta-1)^{3} \beta^{4}\{-16384+5 \beta[6144+\beta(-2976+125 \beta)]\} .
\end{aligned}
$$

For $T=0$ this line $T_{R}$ leads to the critical capacity $\alpha(T=0)=4 / 27=0.14814815$, as seen in Figure 1.

Below the thick full line, $T_{c}$, the retrieval states become globally stable. At this point we remark that, compared with the standard Hopfield model, there is no reentrance behaviour in the transitions since the spherical model is (marginally) replica symmetric. Furthermore, the region of global stability for the retrieval solutions in the spherical model is much larger.

\section{Macroscopic Dynamics.}

In this section we study the dynamics of the model. Since the neurons are continuous, we assume the relaxational dynamics to be given by the set of Langevin equations

$$
\frac{\partial \sigma_{i}(t)}{\partial t}=-\mu(t) \sigma_{i}(t)-\frac{\delta \mathcal{H}[\boldsymbol{\sigma}]}{\delta \sigma_{i}(t)}+\eta_{i}(t)+\theta_{i}(t)
$$


where the first term on the rhs controls the fluctuations of the neurons, $\eta_{i}(t)$ is gaussian noise with as first two moments

$$
\left\langle\eta_{i}(t)\right\rangle=0, \quad\left\langle\eta_{i}(t) \eta_{j}\left(t^{\prime}\right)\right\rangle=2 T \delta_{i j} \delta\left(t-t^{\prime}\right)
$$

and $\theta_{i}(t)$ is an external perturbation field. We discuss this dynamics using the generating functional approach [12, 13. In a straightforward way the following generating functional is introduced

$$
\mathcal{Z}[\boldsymbol{\psi}]=\int \mathbf{D}[\boldsymbol{\sigma}, \widehat{\boldsymbol{\sigma}}] \exp \left[\sum_{i=1}^{N} \int d t \psi_{i}(t) i \sigma_{i}(t)+A[\boldsymbol{\sigma}, \widehat{\boldsymbol{\sigma}}]\right]
$$

with the action $A[\boldsymbol{\sigma}, \widehat{\boldsymbol{\sigma}}]$ given by

$$
A[\boldsymbol{\sigma}, \widehat{\boldsymbol{\sigma}}]=\sum_{i=1}^{N} \int d t\left\{-T \widehat{\sigma}_{i}^{2}(t)+i \widehat{\sigma}_{i}(t)\left(\frac{\partial \sigma_{i}(t)}{\partial t}+\mu(t) \sigma_{i}(t)-\theta_{i}(t)+\frac{\delta \mathcal{H}[\boldsymbol{\sigma}]}{\delta \sigma_{i}(t)}\right)\right\}
$$

where the $\psi_{i}(t)$ are the generating fields, $\widehat{\sigma}_{i}(t)$ are conjugate variables and $\mathbf{D}[\boldsymbol{\sigma}, \widehat{\boldsymbol{\sigma}}]$ is the measure in path space. From this expression all physical quantities of interest can be computed by taking derivatives with respect to the generating and the external perturbation fields. Assuming that the initial neuron configuration is correlated with only one pattern, i.e. the condensed one, and averaging over the disorder we arrive, after some algebra, at the following effective single site dynamics in the thermodynamic limit

$$
\frac{\partial \sigma(t)}{\partial t}=-\mu(t) \sigma(t)+\left[m(t)+u_{0} m^{3}(t)\right] \xi^{1}+\theta(t)+\alpha \int d t^{\prime}\left[(\mathbb{I}-\mathbf{G})^{-1} \mathbf{G}\right]\left(t, t^{\prime}\right) \sigma\left(t^{\prime}\right)+\phi(t)
$$

where $\phi$ is a colored noise with mean value and variance

$$
\langle\phi(t)\rangle_{\star}=0, \quad\left\langle\phi(t) \phi\left(t^{\prime}\right)\right\rangle_{\star}=2 T \delta\left(t-t^{\prime}\right)+\alpha[\mathbb{I}-\mathbf{G}]^{-1} \mathbf{C}\left[\mathbf{I}-\mathbf{G}^{\dagger}\right]^{-1}\left(t, t^{\prime}\right) .
$$

In this expression $m(t)$ is the retrieval overlap

$$
m(t)=\left\langle\left\langle\xi^{1}\langle\sigma(t)\rangle_{\star}\right\rangle\right\rangle_{\xi^{1}}
$$

and the matrices $\mathbf{C}$ and $\mathbf{G}$ are the dynamical order parameters of the problem, i.e. the correlation and response functions

$$
C\left(t, t^{\prime}\right)=\left\langle\left\langle\left\langle\sigma(t) \sigma\left(t^{\prime}\right)\right\rangle_{\star}\right\rangle\right\rangle_{\xi^{1}}, \quad G\left(t, t^{\prime}\right)=\frac{\partial}{\partial \theta\left(t^{\prime}\right)}\left\langle\left\langle\langle\sigma(t)\rangle_{\star}\right\rangle\right\rangle_{\xi^{1}}
$$


with $\langle\langle\cdots\rangle\rangle_{\xi^{1}}$ the average over the condensed pattern and $\langle\cdots\rangle_{\star}$ the average over the effective noise $\phi$. At this point we remark that in the course of the calculation we see explicitly that the quartic term in the Hamiltonian gives no extensive noise contribution.

Taking the perturbation field $\theta(t)$ to zero, using the causality properties of the response function and the spherical constraint, $C(t, t)=1$, it is possible to write down a closed set of equations for the macroscopic observables specifying the dynamics

$$
\begin{aligned}
\left(\frac{\partial}{\partial t}+\mu(t)\right) m(t) & =\left[m(t)+u_{0} m^{3}(t)\right]+\alpha \int_{-\infty}^{t} d t^{\prime} R\left(t, t^{\prime}\right) m\left(t^{\prime}\right) \\
\left(\frac{\partial}{\partial t}+\mu(t)\right) G\left(t, t^{\prime}\right) & =\delta\left(t-t^{\prime}\right)+\alpha \int_{t^{\prime}}^{t} d t_{1} R\left(t, t_{1}\right) G\left(t_{1}, t^{\prime}\right) \\
\left(\frac{\partial}{\partial t}+\mu(t)\right) C\left(t, t^{\prime}\right) & =2 T G\left(t^{\prime}, t\right)+\alpha \int_{-\infty}^{t^{\prime}} d t_{1} S\left(t, t_{1}\right) G\left(t^{\prime}, t_{1}\right) \\
& +\left[m(t)+u_{0} m^{3}(t)\right] m\left(t^{\prime}\right)+\alpha \int_{-\infty}^{t} d t_{1} R\left(t, t_{1}\right) C\left(t^{\prime}, t_{1}\right)
\end{aligned}
$$

where we have defined the effective correlation function, $S\left(t, t^{\prime}\right)$, and response function, $R\left(t, t^{\prime}\right)$, as

$$
S\left(t, t^{\prime}\right)=[\mathbf{I}-\mathbf{G}]^{-1} \mathbf{C}\left[\mathbf{I}-\mathbf{G}^{\dagger}\right]^{-1}\left(t, t^{\prime}\right), \quad R\left(t, t^{\prime}\right)=\left[(\mathbf{I}-\mathbf{G})^{-1} \mathbf{G}\right]\left(t, t^{\prime}\right) .
$$

In order to obtain the stationary state from the equations (29) we assume that, close to equilibrium the order parameters become time translation invariant meaning that the one-time quantities become time independent and the two-time quantities satisfy $C\left(t, t^{\prime}\right)=C\left(t-t^{\prime}\right), G\left(t, t^{\prime}\right)=G\left(t-t^{\prime}\right)$ (and similarly for $R$ and $S$ ). Time translation invariance holds when the system is ergodic. Then the correlation and response functions are related through the fluctuation-dissipation theorem (FDT) [3], 4]

$$
\beta \partial_{\tau} C(\tau)=G(-\tau)-G(\tau), \quad \beta \partial_{\tau} S(\tau)=R(-\tau)-R(\tau)
$$

with $\tau=t-t^{\prime}$, the initial time $-\infty$ and $\partial / \partial \tau$ denoted by $\partial_{\tau}$. With these assumptions we can write the evolution equation for the correlation function in the following way

$$
\begin{aligned}
\left(\partial_{\tau}+\right. & \mu-\alpha \beta[1-S(\tau)]) C(\tau)+\alpha \beta \int_{0}^{\tau} d t^{\prime}\left[S\left(\tau-t^{\prime}\right)-S(\tau)\right] \partial_{t^{\prime}} C\left(t^{\prime}\right) \\
& =\left[m+u_{0} m^{3}\right] m+\alpha \int_{0}^{\infty} d t^{\prime}\left[R\left(t^{\prime}+\tau\right) C\left(t^{\prime}\right)+S\left(t^{\prime}+\tau\right) G\left(t^{\prime}\right)\right] .
\end{aligned}
$$


With the conditions $C(0)=1$ and $\left.\beta \partial_{\tau} C(\tau)\right|_{\tau=0}=-1$ we then have for $\tau \rightarrow \infty$

$$
\left[m+u_{0} m^{3}\right] m=\frac{C(\infty)}{\beta[1-C(\infty)]}-\alpha \beta S(\infty)[1-C(\infty)]
$$

Finally, using the evolution equation for $m$ and

$$
\lim _{\tau \rightarrow \infty} C(\tau)=q, \quad \lim _{\tau \rightarrow \infty} S(\tau)=\frac{q}{[1-\beta(1-q)]^{2}}
$$

it is straightforward to arrive at the equilibrium saddle-point equations (17).

At this point we remark that following [3] by starting from the evolution equation for the correlation function written as

$$
\left(\partial_{\tau}+\mu-\alpha \beta[1-S(\tau)]\right) \beta[1-C(\tau)]-\alpha \beta^{2} \int_{0}^{\tau} d t^{\prime}\left[S\left(\tau-t^{\prime}\right)-S(\tau)\right] \partial_{t^{\prime}} C\left(t^{\prime}\right)=1
$$

and using the fact that the dynamics is purely relaxational, and hence, that $\partial_{t} C(t) \leq$ $0, \partial_{t} S(t) \leq 0$, we can derive the following condition for ergodicity

$$
\frac{1}{\beta[1-C(\tau)]}+\alpha \beta-\mu-\mu S(\tau) \geq 0
$$

This inequality seems to indicate that our system is ergodic in the sense of [3].
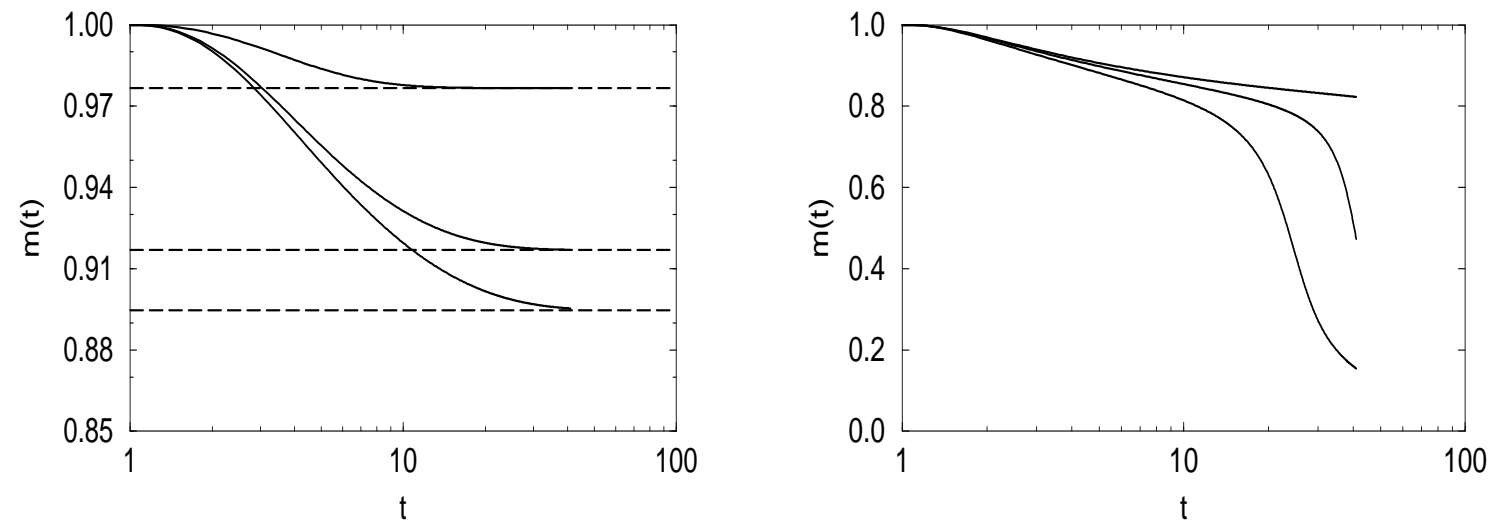

Figure 2: The overlap order parameter $m(t)$ as a function of time at temperature $T=0$ for several values of the capacity: $\alpha=0.041,0112,0127$ from top to bottom on the left and $\alpha=0150,0160,0180$ from top to bottom on the right. The horizontal dashed lines correspond to the stationary value taken from theory. 
In fig. 2 we show some plots of the dynamics (29) obtained by discretizing the equations. Specifically, the overlap order parameter is shown for several values of the loading capacity at temperature zero with $m(0)=1$. The behaviour for nonzero temperature is qualitatively the same. We clearly see that below the critical capacity (figure on the left) the system evolves to the stationary solution, while above the critical capacity (figure on the right) $m(t)$ drops to zero.

\section{Conclusions}

In this work we have presented the spherical version of the Hopfield model. In order to have a retrieval phase a quartic interaction has been introduced which does not destroy the spherical character of the model. The thermodynamic phase diagram is qualitatively the same as the one for the standard Hopfield model, except that there is no re-entrance because the system is shown to be (marginally) replica symmetric. Furthermore, the region of global stability for the retrieval solutions is larger. A closed set of equations is obtained for the Langevin dynamics and the stationary limit is shown to correspond to the thermodynamic results. A numerical calculation

of the evolution of the retrieval overlap order parameter illustrates these findings.

\section{Acknowledgments}

We thank A.C.C. Coolen and R. Kühn for informative discussions. This work has been supported in part by the Fund of Scientific Research, Flanders-Belgium.

\section{References}

[1] T.H. Berlin and M. Kac, Phys. Rev. 86, 821 (1952).

[2] A. Crisanti and H.J. Sommers, Z. Phys. B 87, 341 (1992).

[3] A. Crisanti, H. Horner and H. J. Sommers, Z. Phys. B 92, 271 (1993).

[4] A. Crisanti and F. Ritort, Violation of the fluctuation-dissipation theorem in glassy systems: basic notions and the numerical evidence, to be published in J. Phys. A (Math. Gen.), cond-mat/0212490.

[5] J. Hertz, A. Krogh and R. G. Palmer, Introduction to the Theory of Neural Computation (Addison-Wesley, Redwood City 1995). 
[6] B. Müller, J. Reinhardt and M.T. Strickland, Neural Networks: An Introduction (Springer, Berlin, 1991).

[7] Leticia F. Cugliandolo Lecture notes in Slow Relaxation and non equilibrium dynamics in condensed matter Les Houches Session 77 July 2002, J-L Barrat, J Dalibard, J Kurchan, M V Feigel'man eds. cond-mat/0210312.

[8] J.J. Hopfield, Proc. Nat. Acad. Sci. USA 79, 2554 (1982).

[9] M. Mézard, G. Parisi and M.A. Virasoro, Spin Glass Theory and Beyond (Singapore, World Scientific, 1987).

[10] Th.M. Nieuwenhuizen, Phys. Rev. Lett. 74, 4289 (1995).

[11] D. Amit, H. Gutfreund and H. Sompolinsky, Annals of Physics 173, 30 (1987).

[12] P. C. Martin, E. D. Siggia and H. A. Rose, Phys. Rev. A 8, 423 (1973).

[13] C. De Dominicis, Phys. Rev. B 18, 4913 (1978) 